\title{
Dieta de espécies de Anostomidae (Teleostei, Characiformes) na área de influência do reservatório de Manso, Mato Grosso, Brasil
}

\author{
Graziela Custodio Balassa; Rosemara Fugi; Norma Segatti Hahn \& André Beal Galina
}

Núcleo de Pesquisas em Limnologia, Ictiologia e Aqüicultura (NUPÉLIA), Universidade Estadual de Maringá, Av. Colombo 5790, 87020-900, Maringá, Paraná, Brasil. (rfugi@ nupelia.uem.br)

\begin{abstract}
Diet of Anostomidae species (Teleostei, Characiformes) in the influence area of Manso Reservoir, Mato Grosso, Brazil. The diet of four species of Anostomidae (Leporinus friderici Bloch, 1794, L. striatus Kner, 1858, L. elongatus Valenciennes, 1849 and Leporinus sp.) were investigated in the Manso Reservoir, Mato Grosso State, Brazil. Fish were sampled in three sites: upriver, in the main body of the reservoir, and below the dam. Were analized 276 stomachs. The diet was evaluated using the frequency of ocorrence and volumetric methods. Leporinus friderici showed tendency to herbivory, mainly in the upriver site. In the reservoir and below the dam, it consumed large quantities of fish; Isoptera only in the reservoir. Leporinus striatus and L. elongatus have similar diets, consuming Chironomidae larvae (Diptera), whereas Leporinus sp. was more generalist, feeding similar proportions of vegetable, detritus and insect (Chironomidae and Ephemeroptera). The mouths of the species have different positions: terminal in L. friderici, subterminal in L. elongatus and intermediate in L. striatus and Leporinus sp. The diet overlap values were low, except for L. friderici and Leporinus sp. (0.7) in the upriver, and L. striatus and L. elongatus (0.6) below the dam.
\end{abstract}

KEYWORDS. Leporinus, diet, Manso Reservoir.

\section{INTRODUÇÃO}

A flexibilidade de hábito alimentar é uma característica adaptativa do comportamento animal, uma vez que ambientes naturais variam espacial e temporalmente, sendo que os peixes respondem à baixa disponibilidade alimentar alterando seu comportamento. Em reservatórios recém-formados, alterações no comportamento alimentar dos peixes são esperadas, principalmente em função da incorporação de material terrestre ao sistema aquático (Agostinho et al., 1999) e do incremento da produção em todos os níveis tróficos (O'BRIEN, 1990), que pode alterar temporariamente a disponibilidade dos recursos alimentares. Espera-se que espécies estreitamente relacionadas apresentem, no mínimo, estratégias e táticas comportamentais diferenciadas que permitam a co-existência. Em relação à partilha de recursos alimentares, espécies de peixes congenéricas ou mesmo simpátricas têm mostrado, além de diferenças comportamentais (Agostinho et al., 2003), atributos estruturais altamente desenvolvidos para a tomada do alimento (Fugi \& HaHn, 1991; Fugi et al., 1996; Delariva \& Agostinho, 2001; Fugl et al., 2001). Estes atributos incluem a forma e posição da boca e dos dentes, tipos de rastros branquiais, além da forma e tamanho do intestino (WoOtToN, 1990). Ao contrário dos organismos terrestres, para os quais a segregação de habitat é mais importante, para as comunidades aquáticas a segregação trófica tem se mostrado a mais expressiva (Ross, 1986). Investigações do espectro alimentar, por meio de estudos de conteúdos estomacais, aliadas a análises morfológicas de órgãos envolvidos na tomada do alimento, auxiliam na interpretação da dinâmica e ocupação de habitats por tais espécies.

Foram investigadas quatro das seis espécies de Leporinus Spix, 1829, registradas na área do reservatório de
Manso, Mato Grosso, com o objetivo de esclarecer possíveis diferenças em suas dietas, bem como inferir sobre suas estratégias alimentares em função de atributos estruturais.

\section{MATERIAL E MÉTODOS}

O reservatório de Manso localiza-se no Estado de Mato Grosso, próximo ao Parque Nacional da Chapada dos Guimarães. O Rio Manso, onde foi construído o reservatório, é o principal formador do Rio Cuiabá. Fechado em novembro de 1999, apresentou um rápido enchimento (novembro de 1999 a fevereiro de 2000, fig.1) inundando, além do trecho do rio Manso, à montante da barragem, as porções inferiores dos rios Casca, Palmeiras e Quilombo, totalizando uma área inundada de $427 \mathrm{~km}^{2}$ na cota máxima.

Os peixes utilizados são provenientes de coletas realizadas em onze estações de amostragem, sendo sete no reservatório de Manso (MM-1, MM-2, MM-3, CA-1, CA-2, QU, PA), três à jusante do reservatório (MJ-1, MJ-

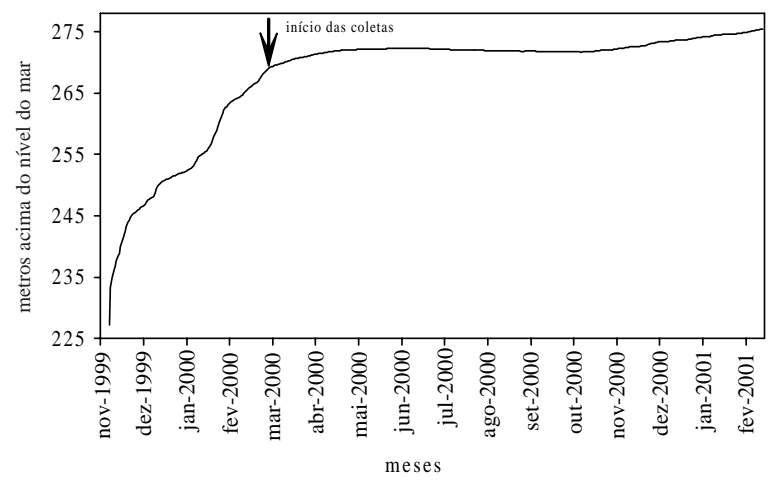

Fig. 1. Variações mensais no nível do reservatório de Manso, Mato Grosso, no período de novembro de 1999 a fevereiro de 2001. 
2, MJ-3), e uma à montante do reservatório (MM-4) (fig. 2). Os peixes foram amostrados utilizando-se redes de espera (malhas 2,$4 ; 3 ; 4 ; 5 ; 6 ; 7 ; 8 ; 9 ; 10 ; 12 ; 14 ; 16 ; 18$ e 30 $\mathrm{cm}$, entre nós opostos), com 10 metros de comprimento por malha. As redes ficaram expostas por 24 horas, com revistas ao amanhecer $(9 \mathrm{~h})$, ao entardecer ( $17 \mathrm{~h})$ e à noite $(23 \mathrm{~h})$. As coletas foram realizadas mensalmente de março de 2000 a fevereiro de 2001. Os peixes foram identificados, pesados (em gramas), medidos (comprimento padrão, Cp, dado em $\mathrm{cm}$ ) e os estômagos retirados e fixados em formalina $4 \%$.

As espécies incluídas nesta análise foram: Leporinus friderici Bloch, 1794, L. striatus Kner, 1858, L. elongatus Valenciennes, 1849 e Leporinus sp. Os exemplares coletados estão depositados na Coleção
Ictiológica, NUPÉLIA, Universidade Estadual de Maringá, Paraná (responsável, C. S. Pavanelli). Leporinus lacustris Campos, 1945 e L. obtusidens (Valenciennes, 1847), também registradas nas coletas, foram raras e, em função do tamanho da amostra, não foram incluídas nas análises da dieta. Foram obtidos dados de variação de tamanho dos peixes analisados e o número de estômagos utilizados (tab. I).

A abundância das espécies foi avaliada por local de coleta (montante, reservatório e jusante), com base na captura por unidade de esforço (CPUE) que foi expressa como sendo o número de indivíduos por $1000 \mathrm{~m}^{2}$ de rede em 24 horas.

No laboratório, os conteúdos estomacais foram examinados, estimando-se a importância dos itens

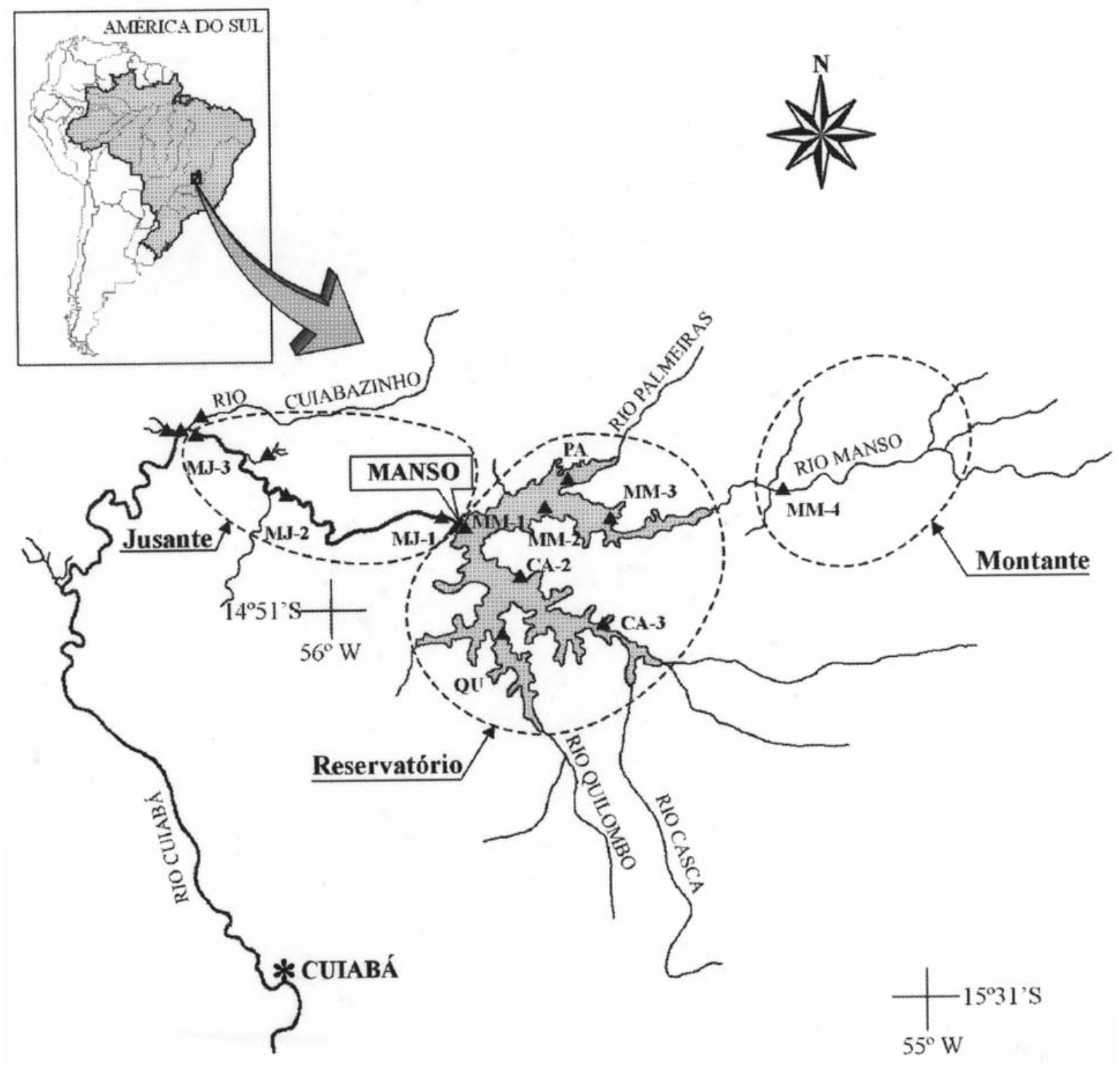

Fig. 2. Localização das estações de amostragem no reservatório de Manso, Mato Grosso, à montante e à jusante. 
alimentares de acordo com a ocorrência e volume. O volume de cada item alimentar foi obtido através do deslocamento da coluna de água, utilizando-se uma bateria de provetas graduadas. Para itens alimentares que apresentaram volume inferior a $0,1 \mathrm{ml}$ foi utilizada uma placa milimetrada, obtendo-se o volume em $\mathrm{mm}^{3}$ e posteriormente transformado em ml (HellawEl \& ABEL, 1971).

A sobreposição da dieta foi calculada usando o Índice de Schoener: $S=1-0,5\left(\sum_{i=1}^{n}\left|\mathrm{P}_{x i}-\mathrm{P}_{y i}\right|\right)$ onde, $\mathrm{P}_{x i}$ e $\mathrm{P}_{y i}$ são as proporções (volume) do item alimentar $i$ usado pelas espécies $x$ e $y$, e $n$ é o número total de itens alimentares. Este índice varia entre 0 e 1, e a sobreposição é considerada significativa quando o valor for igual ou superior a 0,6 (ZARET \& RAND, 1971; WALLACE, 1981).

Tabela I. Número de exemplares, por local de coleta, na área do reservatório de Manso, Mato Grosso, e variação do comprimento padrão (Cp) das quatro espécies de Leporinus analisadas para o estudo da dieta (M, montante; R, reservatório; J, jusante).

\begin{tabular}{lrrrrr}
\hline & & \multicolumn{3}{c}{ Locais de coleta } & \\
\cline { 3 - 5 } Espécies & $\mathrm{Cp}(\mathrm{cm})$ & $\mathrm{M}$ & $\mathrm{R}$ & $\mathrm{J}$ & Total \\
\hline L. friderici & $6,4-32,5$ & 21 & 151 & 16 & 188 \\
L. elongatus & $15,0-36,0$ & & 18 & 6 & 24 \\
L. striatus & $6,5-11,0$ & 5 & 17 & 28 & 50 \\
Leporinus $\mathrm{sp}$. & $7,3-10,6$ & 14 & & & 14 \\
\hline
\end{tabular}

\section{RESULTADOS E DISCUSSÃO}

Foram registradas durante as coletas seis espécies do gênero Leporinus, sendo que L. friderici destacouse como a mais abundante, principalmente no reservatório e à montante (figs. 3,4 ); L. striatus e Leporinus sp., embora menos abundantes que L. friderici, foram relativamente bem representadas nas capturas, a primeira principalmente à jusante e a segunda à montante do reservatório. Leporinus elongatus, embora pouco representativa, ocorreu na mesma proporção nos três locais; L. lacustris e L. obtusidens foram espécies raras nas capturas. As diferenças nas proporções e distribuição

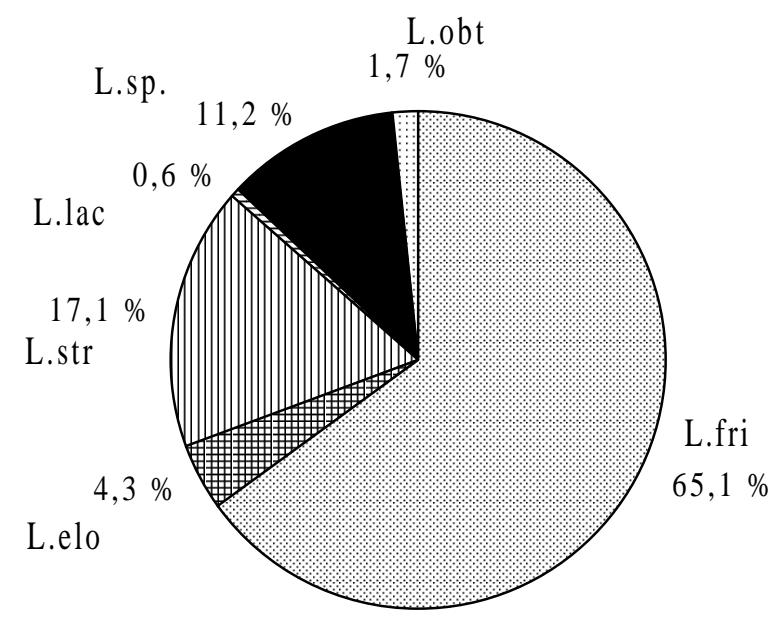

Fig. 3. Frequiência relativa das espécies de Leporinus na área do reservatório de Manso, Mato Grosso. (L.fri, L. friderici; L.elo, L. elongatus; L.str, L. striatus; L.obt, L. obtusidens; L.lac, L. lacustris; L.sp., Leporinus sp.).

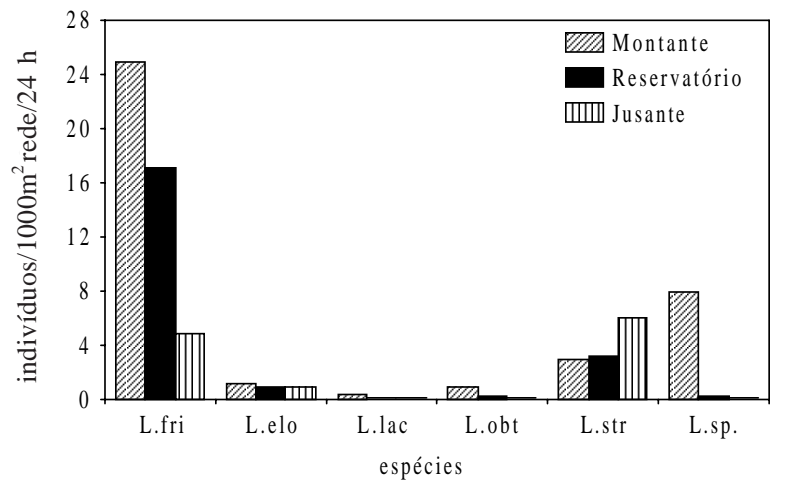

Fig. 4. Distribuição, por local de coleta, com base na captura por unidade de esforço, das espécies de Leporinus na área do reservatório de Manso, Mato Grosso (L.fri, L. friderici; L.elo, L. elongatus; L.str, L. striatus; L.obt, L. obtusidens; L.lac, L. lacustris; L.sp., Leporinus sp.).

destas espécies, nos diferentes locais, podem ser uma ocorrência natural, como parece ser o caso de Leporinus sp., capturada quase que exclusivamente no ambiente fluvial (montante), e/ou o resultado da acomodação no ambiente represado, como verificado para $L$. friderici. Esta espécie, embora realize migrações longitudinais (VAZZOLER, 1996), foi o anostomídeo mais abundante no reservatório e tem importante participação na pesca em reservatórios da bacia do Rio Paraná (Agostinho, 1994). Nos reservatórios de Corumbá, Goiás, e Rosana, São Paulo/Paraná, não foram verificadas alterações relevantes na abundância de L. friderici antes e após a construção da barragem (AgostinHo et al., 1999).

As quatro espécies analisadas apresentaram amplo espectro alimentar, sendo a dieta representada por vegetais, detritos, insetos de diferentes ordens, invertebrados aquáticos e peixes (tab. II). A onivoria de espécies de Leporinus tem sido amplamente enfatizada na literatura (ANDRIAN et al., 1994; HAHN et al., 1998; DURÃEs et al., 2001).

Para L. friderici, a alta freqüência e abundância de vegetais nos estômagos de exemplares capturados à montante, ambiente não impactado pela formação do reservatório, indica uma tendência à herbivoria, hábito este também comentado por ANDRIAN et al. (1994) e HAHN et al. (1997) para exemplares da planície de inundação do alto Rio Paraná. No reservatório de Manso e à jusante, a espécie consumiu peixes em proporções relevantes, fato constatado também no reservatório de Nova Ponte, Minas Gerais (DurÃEs et al., 2001) e Serra da Mesa, Goiás (Albrecht \& CARAmaschi, 2003). O fato de o alimento ingerido ser representado por pedaços de musculatura de peixes sugere que esta espécie comporta-se ocasionalmente como necrófaga. O consumo de peixes por L. friderici evidencia sua estratégia alimentar oportunista (sensu GERKING, 1994), uma vez que explosões populacionais de espécies de peixes forrageiros são esperadas no início da formação de um reservatório (Agostinho et al., 1999). Deve-se considerar ainda que, durante o enchimento, o reservatório pode ser submetido a condições de anoxia afetando negativamente a sobrevivência dos peixes (Agostinho et al., 1999), tornando-os um alimento disponível e de fácil acesso. $\mathrm{O}$ caráter oportunista é também expresso pelo consumo de Isoptera (quase que exclusivo desta espécie), o qual não 
Tabela II. Composição, em percentagem de ocorrência (o) e volume (v), da dieta de quatro espécies de Leporinus na área do reservatório de Manso, Mato Grosso (L.fri, L. friderici; L.elo, L. elongatus; L.str, L. striatus; L.sp., Leporinus sp.; M, montante; R, reservatório; J, jusante).

\begin{tabular}{|c|c|c|c|c|c|c|c|c|c|c|c|c|c|c|c|c|c|c|}
\hline \multirow{3}{*}{ Itens } & \multicolumn{6}{|c|}{ L.fri } & \multicolumn{4}{|c|}{ L.elo } & \multicolumn{6}{|c|}{ L.str } & \multirow{2}{*}{\multicolumn{2}{|c|}{$\begin{array}{c}\text { L.sp. } \\
\mathrm{M}\end{array}$}} \\
\hline & \multicolumn{2}{|c|}{$\mathrm{M}$} & \multicolumn{2}{|c|}{$\mathrm{R}$} & \multicolumn{2}{|c|}{$\mathrm{J}$} & \multicolumn{2}{|c|}{$\mathrm{R}$} & \multicolumn{2}{|c|}{$\mathrm{J}$} & \multicolumn{2}{|c|}{ M } & \multicolumn{2}{|c|}{$\mathrm{R}$} & \multicolumn{2}{|c|}{$\mathrm{J}$} & & \\
\hline & o & $\mathrm{V}$ & 0 & $\mathrm{v}$ & 0 & $\mathrm{v}$ & o & $\mathrm{v}$ & o & $\mathrm{v}$ & o & $\mathrm{v}$ & o & $\mathrm{v}$ & 0 & $\mathrm{v}$ & o & $\mathrm{v}$ \\
\hline Vegetal & 38,9 & 66,5 & 16,8 & 21,3 & 43,5 & 37,6 & 4,2 & 1,3 & 16,7 & 24,0 & 14,3 & 23,3 & 8,3 & 20,5 & 27,7 & 18,8 & 26,3 & 51,3 \\
\hline Alga & 2,7 & 0,7 & 3,0 & 8,7 & 8,7 & 20,5 & 2,1 & 2,3 & 16,7 & 35,9 & & & 8,3 & 5,0 & 21,3 & 34,7 & & \\
\hline Peixe & 16,7 & 9,3 & 16,8 & 32,4 & 34,8 & 41,6 & & & & & & & & & & & 31,6 & 3,9 \\
\hline Diptera & 16,7 & 1,0 & 22,4 & 12,7 & 4,3 & 0,1 & 29,8 & 45,2 & 41,7 & 31,5 & 14,3 & 0,9 & 54,2 & 73,0 & 14,9 & 1,1 & & \\
\hline Isoptera & & & 16,2 & 17,9 & & & 2,1 & 8,7 & & & & & 4,2 & 0,1 & & & & \\
\hline Hymenoptera & 8,3 & 0,2 & 6,6 & 1,9 & 4,3 & 0,1 & & & & & 14,3 & 0,9 & & & & & & \\
\hline Lepidoptera & 2,8 & 2,3 & 0,7 & 0,9 & & & & & & & & & & & & & & \\
\hline Trichoptera & 5,6 & 3,1 & 2,3 & 0,8 & & & 6,4 & 5,2 & & & 14,3 & 9,8 & 8,3 & 0,9 & 6,4 & 1,8 & & \\
\hline Odonata & & & 2,3 & 1,9 & & & 14,9 & 16,6 & & & & & & & & & & \\
\hline Ephemeroptera & & & & & & & & & & & & & & & & & 15,8 & 26,4 \\
\hline Conchostraca & & & & & & & 19,1 & 16,8 & & & & & & & & & & \\
\hline Invertebrados & & & 11,2 & 1,0 & & & 6,4 & 0,1 & 8,3 & 4,5 & 14,3 & 0,5 & 8,3 & 0,5 & 2,1 & 0,9 & 5,3 & 0,1 \\
\hline Detrito & 8,3 & 16,9 & 1,6 & 0,5 & 4,3 & 0,2 & 14,9 & 3,8 & 16,1 & 4,1 & 28,5 & 64,6 & 8,3 & & 27,6 & 42,6 & 21,0 & 18,2 \\
\hline
\end{tabular}

\section{Leporinus friderici}

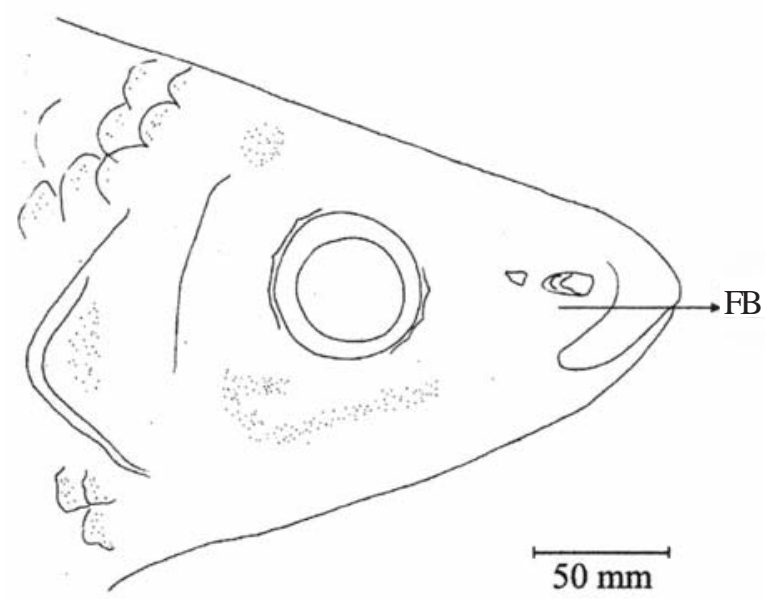

Leporinus striatus

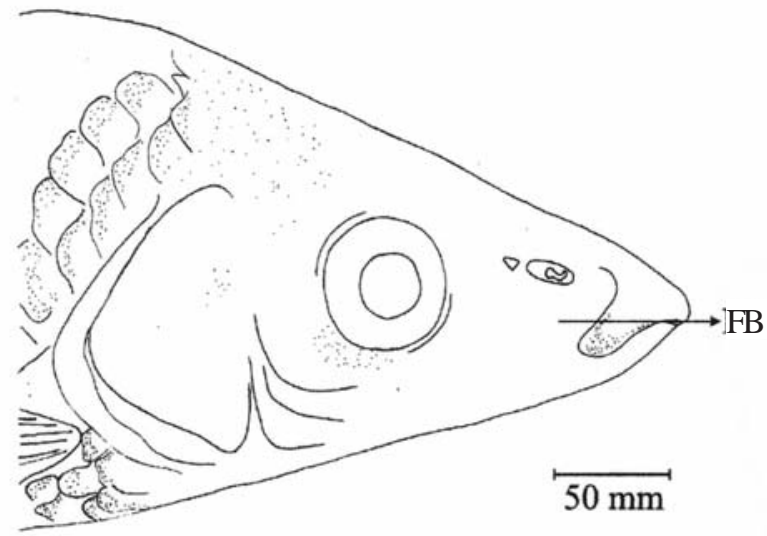

\section{Leporinus elongatus}

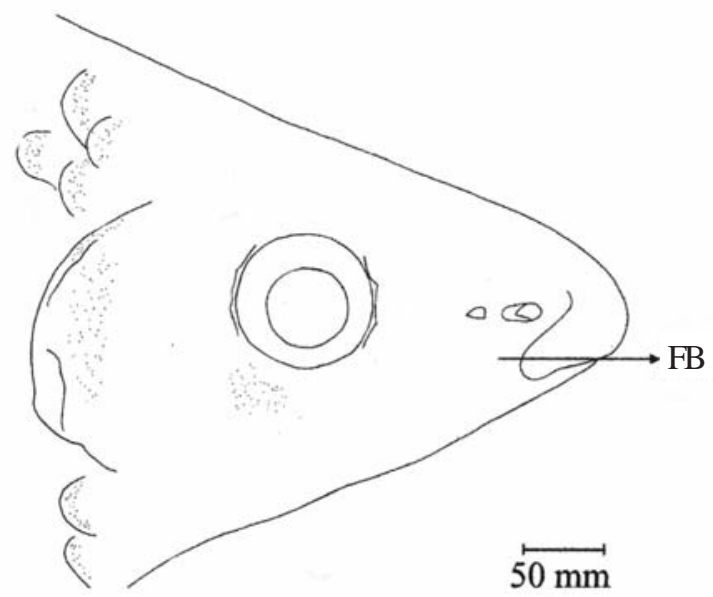

Leporinus sp.

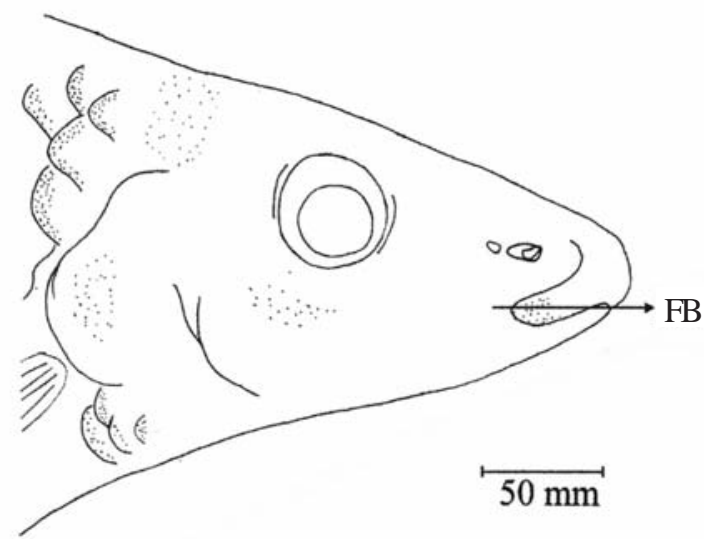

Fig. 5. Posição da boca para as quatro espécies de Leporinus coligidas no reservatório de Manso, Mato Grosso (FB, fenda bucal). 
foi registrado na dieta de exemplares capturados à montante e à jusante. O início do enchimento do reservatório de Manso foi marcado por um rápido alagamento (novembro de 1999 a fevereiro de 2000), inundando grandes extensões de terra e incorporando, conseqüentemente, cupinzeiros de áreas circunvizinhas. O consumo de Isoptera foi verificado também nos reservatórios de Nova Ponte, Minas Gerais (DuRÃEs et al., 2001) e Serra da Mesa, Goiás (Albrecht \& CARAMASCHI, 2003) para esta espécie.

Leporinus striatus e L. elongatus apresentaram dietas mais similares quando se considera o alimento principal. O elevado consumo de larvas de Chironomidae (Diptera), principalmente no reservatório, para a primeira, e reservatório e jusante, para a segunda espécie indica que ambas exploram o sedimento. O elevado consumo de Chironomidae está associado à grande produtividade de invertebrados verificada no início da formação de um reservatório (O'BRIEN, 1990), que se tornam um recurso abundante para os peixes. Esses insetos estão entre os primeiros a colonizar áreas recentemente alagadas (DAVIES, 1976). Outra evidência do uso de substratos como habitat de forrageamento por essas espécies foi o consumo de massas de algas filamentosas, à jusante do reservatório, que colonizam o fundo, onde provavelmente proliferaram após o fechamento da barragem, em decorrência da diminuição da vazão. Para Leporinus sp., o reduzido número de estômagos analisados não permitiu inferências mais conclusivas, porém esta espécie mostrou-se a mais onívora, consumindo proporções semelhantes de vegetais e insetos (larvas de Chironomidae e Ephemeroptera), além de detritos.

A posição da boca apresenta-se diferente para as quatro espécies de Leporinus (fig. 5), variando de um gradiente terminal a subterminal. Leporinus friderici apresenta boca em posição terminal, enquanto em $L$. elongatus essa é mais inferior, com lábio superior muito desenvolvido. Leporinus striatus e Leporinus sp. apresentam boca subterminal, caracterizando uma posição intermediária entre as outras duas espécies.

Embora a maioria dos peixes apresente elevada plasticidade alimentar, essa pode ser limitada, dentre outros fatores, pela morfologia trófica (GERKING, 1994). Apesar de SANTOS \& Rosa (1998) sugerirem que a posição da boca, entre os anostomídeos, não seja sempre uma indicação do hábito alimentar, no caso das espécies aqui estudadas, observou-se que, mesmo se comportando como oportunistas, a posição da boca representa uma característica conservativa, se não limitando a captura do alimento, facilitando o consumo de alimento temporariamente disponível. Esta relação pode ser observada pelo elevado consumo de Isoptera, por $L$. friderici, cuja boca terminal facilita a captura de insetos à deriva, o que seria mais difícil para espécies com boca subterminal, como é o caso das demais espécies. O predomínio de Chironomidae, algas filamentosas e detrito na dieta de L. striatus e L. elongatus reforça essa suposição, uma vez que a boca subterminal facilita a alimentação no fundo.

A análise da sobreposição da dieta das espécies mostrou, para a maioria dos pares analisados, valores baixos (inferiores a 0,6 ), com exceção de $L$. friderici $e$
Leporinus sp. $(0,7)$ à montante, e entre $L$. striatus e $L$. elongatus $(0,6)$ à jusante (fig. 6). Estes valores estão relacionados ao elevado consumo de vegetais para o primeiro par e de algas filamentosas para o segundo. A baixa sobreposição entre a dieta das espécies está associada à elevada capacidade desses peixes em utilizar de forma diferenciada os recursos alimentares temporariamente disponíveis, como insetos incorporados do ambiente terrestre, larvas de insetos (principalmente Chironomidae) e algas filamentosas que colonizam rapidamente o ambiente impactado. A posição da boca auxilia a segregação, porém, a abundância do alimento na fase inicial de represamento e a plasticidade trófica dessas espécies também contribuem para a coexistência.

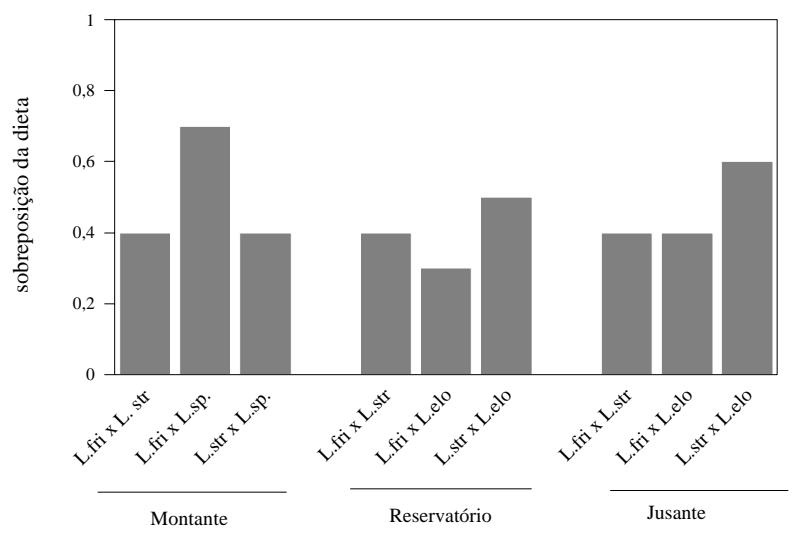

Fig. 6. Variação espacial na sobreposição da dieta entre as quatro espécies de Leporinus no reservatório de Manso, à montante e à jusante (L.fri, L. friderici; L.elo, L. elongatus; L.str, L .striatus; L.sp., Leporinus sp.).

Agradecimentos. Ao NUPÉLIA pelo apoio logístico e a Furnas Centrais Elétricas pelo suporte financeiro.

\section{REFERÊNCIAS BIBLIOGRÁFICAS}

Agostinho, A. A. 1994. Pesquisas, monitoramento e manejo da fauna aquática em empreendimentos hidrelétricos. In: COMASE; ELETROBRÁS. Seminário sobre Fauna Aquática e o Setor Elétrico Brasileiro. Rio de Janeiro. p.38-58. (Fundamentos, cad. 1).

Agostinho, C. S.; Hahn, N. S. \& Marques, E. E. 2003. Patterns of food resources use by two congeneric species of piranhas (Serrasalmus) on the upper Parana river floodplain. Brazilian Journal of Biology, São Carlos, 63(2):177182 .

Agostinho, A. A.; Miranda, L. E. et al. 1999. Patterns of colonization in neotropical reservoirs, and prognoses on aging. In: Tundisi, J. G. \& Straskraba, M. eds. Theoretical reservoir ecology and its applications. São Carlos, Backhuys Publishers. p.227-265.

Albrecht, M. P. \& Caramaschi, E. P. 2003. Feeding ecology of Leporinus friderici (Teleostei; Anostomidae) in the upper Tocantins river, central Brazil, before and after installation of a hydroelectric plant. Studies on Neotropical Fauna and Environment, Lisse, 38(1):3340 .

Andrian, I. F; Dória, C. R. C. et al. 1994. Espectro alimentar e similaridade na composição da dieta de quatro espécies de Leporinus (Characiformes,

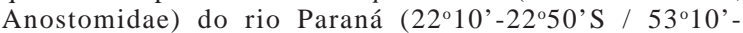
534’ W), Brasil. Revista UNIMAR, Maringá, 16:97106. 
Davies, B. R. 1976. The dispersal of Chironomidae larvae: a review. Journal Entomological Society of Southern Africa, Pretoria, 39:39-62.

Delariva, R. L. \& Agostinho, A. A. 2001. Relationship between morphology and diets of six neotropical loricariids. Journal of Fish Biology, London, 58:832-847.

Durães, R.; Pompeu, P. S. \& Godinho, A. A. L. 2001. Alimentação de quatro espécies de Leporinus (Characiformes, Anostomidae) durante a formação de um reservatório no sudeste do Brasil. Iheringia, Sér. Zool., Porto Alegre, 90: $183-191$.

Fugi, R. \& Hahn, N. S. 1991. Espectro alimentar e relações morfológicas com o aparelho digestivo de três espécies de peixes comedores de fundo do rio Paraná, Brasil. Revista Brasileira de Biologia, Rio de Janeiro, 51(4):873-879.

Fugi, R.; Agostinho, A. A. \& Hahn, N. S. 2001. Trophic morphology of five benthic-feeding fish species of a tropical floodplain. Revista Brasileira de Biologia, São Carlos, 61(1):27-33.

Fugi, R.; Hahn, N. S. \& Agostinho, A. A. 1996. Feeding styles of five species of bottom feeding fishes of the high Paraná river. Environmental Biology of Fishes, Dordrecht, 46:297307.

Gerking, S. D. 1994. Feeding ecology of fish. San Diego, Academic. 416p.

Hahn, N. S.; Agostinho, A. A. et al. 1998. Estrutura trófica da ictiofauna do reservatório de Itaipu (Paraná-Brasil) nos primeiros anos de sua formação. Interciência, Caracas, 23(5):299-305.
Hahn, N. S.; Andrian, I. F. et al. 1997. Ecologia trófica. In: Vazzoler, A. E. A. M.; Agostinho, A. A. \& Hahn, N. S. eds. A planície de inundação do alto rio Paraná: aspectos físicos, biológicos e socioeconômicos. Maringá, Universidade Estadual de Maringá. p. 209-228.

Hellawell, J. M. \& ABel, R. 1971. A rapid volumetric method for the analysis of the food of fishes. Journal of Fish Biology, London, 3:29-37.

O'Brien, W. J. 1990. Perspectives on fish in reservoir limnology. In: Thornton, K. W.; Kimmel, B. L.\& Payne, F. E. eds. Reservoir limnology: ecological perspectives. New York, John Wiley. p.209-225.

Ross, S. T. 1986. Resource partitioning in fish assemblages: a review of field studies. Copeia, Lawrence, 2:352-388.

Santos G. M. \& Rosa P. S. 1998. Alimentação de Anostomus ternetzi e Synaptolaemus cingulatus, duas espécies de peixes amazônicos com boca superior. Revista Brasileira de Biologia, Rio de Janeiro, São Carlos, 58:255-262.

VAzzoler, A. E. A. DE M. 1996. Biologia da reprodução de peixes teleósteos: teoria e prática. Maringá, Universidade Estadual de Maringá. 169p.

Wallace, R. K., JR. 1981. An assessment of diet-overlap indexs. Transactions of the American Fisheries Society, Bethesda, 110:72-76.

Wootton, R. J. 1990. Ecology of teleost fishes. London, Chapman \& Hall. 440p.

ZARET, N. T. \& RAND, A. S. 1971. Competition in tropical stream fishes: support for the competitive exclusion principle. Ecology, New York, 52(2):336-342. 